\title{
Innate Immune Response to House Dust Mite Allergens in Allergic Asthma
}

\author{
Winna Soleha1, Febriana Catur Iswanti² \\ ${ }^{1}$ Master's Programme in Biomedical Sciences, Faculty of Medicine, Universitas Indonesia, Jakarta, Indonesia \\ ${ }^{2}$ Department of Biochemistry and Molecular Biology, Faculty of Medicine, Universitas Indonesia, Jakarta, Indonesia
}

Asthma is a major health problem and one of the leading causes of death in the world. The prevalence of asthma in Indonesia is high, with a recurrence $>50 \%$. Allergic sensitization in asthma is mainly caused by house dust mite (HDM) allergens, both from the mite's body and its contaminants (e.g., lipopolysaccharides). HDM allergens stimulate several pathways in the innate immune response based on the HDM allergen groups that sensitize them. The innate immune response to HDM allergen exposure occurs when pattern recognition receptors (PRRs) recognizes the allergen, thereby stimulating respiratory epithelial cells to release cytokines, namely, thymic stromal lymphopoietin (TSLP), interleukin-25 (IL -25), and IL33. The release of IL-25 and IL-33 activates group 2 innate lymphoid cells (ILC2) to release Th2-type cytokines (i.e., IL-5 and IL-13), resulting in allergic airway inflammation via IgE secretion by B cells, recruitment of eosinophils, and respiratory tract remodeling. Dendritic cells induce an adaptive immune response through Th2 activation in the sensitization and effector phases. Other mediators that contributed to the innate immune response include C-C motif chemokine ligand 20 (CCL20) and granulocyte-macrophage colony-stimulating factor (GM-CSF). A deeper understanding of the components and mechanisms involved in innate immunity against HDM allergens creates the potential to develop alternative therapeutic targets for allergic asthma treatment.

Keywords: house dust mite allergens, innate immunity, allergic asthma, respiratory epithelium, inflammatory cytokines

\section{Introduction}

Asthma has become a major health concern because it affects more than 300 million people worldwide; this figure is estimated to rise to 400 million by 2025 . Asthma is ranked in the top five diseases causing the death of 5-30\% of the world's population. ${ }^{1,2}$ According to the World Health
Organization (WHO), in 2016 as much as 235 million people worldwide suffer from asthma. Many asthmatics are underdiagnosed, resulting in a mortality rate of $>80 \%$ in developing countries.

Global Initiative for Asthma (GINA), asthma incidence in various countries ranged from $1 \%$ to $18 \% .^{3}$ In 2011 , Indonesia's Ministry of Health stated that asthma was ranked

Date of submission: July 24, 2021

Last Revised: September 20, 2021

Accepted for publication: September 21, 2021

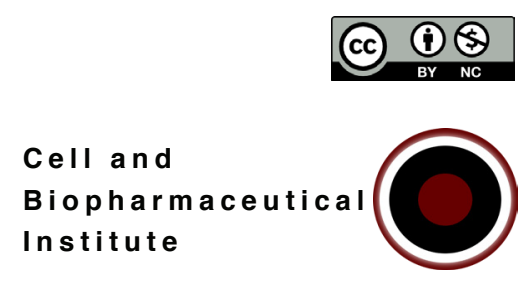

Corresponding Author:

Febriana Catur Iswanti

Department of Biochemistry and Molecular Biology

Faculty of Medicine, Universitas Indonesia

Jl. Salemba Raya No.6, Jakarta, Indonesia

Email: febriana.iswanti@ui.ac.id 
in the top ten causes of death in Indonesia. Furthermore, the mortality rate of asthma is estimated to increase by roughly $20 \%$ in the next ten years if not controlled appropriately. Asthma incidence in Indonesia was $2.4 \%$ in 2018, and the percentage of asthma recurrence increased to $57.5 \%$ in the past year. ${ }^{3,4}$

Asthma is caused by atopy (heredity; 30\%) and nonatopic factors $(70 \%){ }^{1}$ House dust mite (HDM) allergy is the most common cause of allergic sensitization in asthma patients and affects millions of people worldwide. ${ }^{5,6}$ Approximately $85 \%$ of asthmatics are caused by HDM allergens ${ }^{7}$, and $90 \%$ of asthmatic patients in Indonesia are more susceptible to HDM allergens. ${ }^{8}$ HDMs are a significant source of allergens, causing allergic rhinitis and allergic asthma. ${ }^{9}$ More than 35 groups of Dermatophagoides allergens have been identified. Group $1(\operatorname{Der} p 1, \operatorname{Der} f 1)$ and Group $2(\operatorname{Der} p 2, \operatorname{Der} f 2)$ are the main HDM allergen groups. Sensitization to these groups is associated with morbidity in HDM allergic disease. ${ }^{5}$ It is vital to understand the immune response to and pathophysiology of HDM allergens to potentially reveal new targets for therapeutic intervention as the innate immune response may be involved in the development and protection against asthma. ${ }^{10,11}$ Therefore, further research is needed regarding new targets for allergic asthma therapy.

\section{Immune response: innate and adaptive}

Immunity is protection against disease, especially infectious diseases. The cells and molecules that play a role in immunity are defined as the immune system. The immune system's coordinated response to a foreign substance is known as the immune response. The immune response serves a physiological function against infectious agents, such as microbes. Additionally, non-infectious foreign substances and damaged cell products can also trigger an immune response. ${ }^{12}$

Innate immunity is the body's initial defense mechanism against infectious microbes and is mediated by phagocytes, including macrophages and dendritic cells (DCs). A natural immune response already exists in the body before infection occurs. ${ }^{12,13}$ Innate immunity comprises almost all tissues, especially the skin's surface barrier and the respiratory and digestive tracts' mucous membranes. The innate immune system can detect various conditions, such as infection, tissue damage, and genotoxic stress through a receptor encoded in germline DNA, pattern recognition receptors (PRRs). ${ }^{14,15}$ Innate immune receptors bind to pathogen-associated molecular pattern (PAMP) components on microbes as ligands. ${ }^{15}$ The innate immune response can be mediated by phagocytic and cytotoxic mechanisms or the secretion of certain factors, such as antimicrobial peptide (AMP), complement factors, cytokines/chemokines, chitinases, acute phase proteins, proteases, and other molecules. ${ }^{14}$ The innate immune response can then activate and regulate the adaptive immune system. ${ }^{11}$

In contrast to the innate immune response, the adaptive immune response is formed due to exposure to infectious agents and possesses increased resistance and specificity due to its ability to recognize antigens using $\mathrm{T}$ cell receptors. ${ }^{12,15}$ The adaptive immune response is the body's immune response that adapts to exposure to infectious agents (i.e., microbial and non-microbial antigens). ${ }^{12}$ The adaptive immune response is triggered by changes in the cellular environment due to pathogenic antigen accumulation preceded by innate immune mechanisms. ${ }^{16}$

\section{Allergy and allergic asthma}

The term 'allergy' is often used to describe the pathological condition of hypersensitivity. Hypersensitivity reactions can be divided into fast or delayed types based on the time required from antigen exposure until clinical symptoms appear. The classification of hypersensitivity reactions is divided into four types according to Gell and Coombs: types I, II, and III, which are antibody-mediated reactions with or without the contribution of the complement system, and type IV, which is cell-mediated reactions. ${ }^{17}$

Type I hypersensitivity, also called immediate hypersensitivity, is IgE (Immunoglobulin E) antibody-mediated hypersensitivity. Allergies are type I hypersensitivity reactions with various clinical manifestations (e.g., anaphylaxis, asthma, urticaria, and hay fever). This is due to the body's production of IgE antibodies that exceed normal limits in response to an allergen. ${ }^{18,19}$

The common symptoms of asthma are shortness of breath, chest tightness, and a prolonged expiratory phase, with possible wheezing and coughing. Allergic asthma is one of the important manifestations of atopy. Atopy is a genetically determined disease associated with IgE sensitization due to environmental allergens. ${ }^{16,19}$ The most significant risk factors for asthma are due to genetic factors combined with environmental influences, including a history of childhood infections and exposure to allergy- 
triggering inhaled substances. These substances include pollutants and environmental allergens (e.g., pollen, animal dander, and house dust mites). ${ }^{10}$ The clinical manifestations of atopy include food allergy, allergic rhinitis, and asthma. ${ }^{19}$ Atopic individuals often develop various allergic diseases to various allergens, for example, atopic eczema. Subjects with atopic eczema, which develops in childhood due to food antigen sensitization, tend to develop allergic rhinitis and allergic asthma later in life. ${ }^{16}$

Elevated IgE levels in atopic individuals are believed to be a consequence of type-2 $\mathrm{T}$ helper cell (Th2) activation and subsequent release of proinflammatory cytokines, including interleukin (IL)-4, IL-5, IL-13, and IL-31. However, asthma does not always develop from allergies. Based on the source of the trigger, asthma is divided into allergic (extrinsic) and non-allergic (intrinsic). In allergic asthma, inhaled airborne allergens that reach the lower respiratory tract can cause chronic allergic inflammation, a clinical manifestation of allergic asthma. Another prominent feature of allergic asthma is the elevation of total IgE levels and antigen-specific IgE (Table 1). ${ }^{19}$

Triggers of asthma are often environmental and found inside and outside the home. Asthma tends to develop in children with a family history of asthma. For asthmatics, every inhaled substance that enters the respiratory tract can trigger asthma recurrence. Environmental factors that trigger asthma can be allergens, including animal dander and house dust mites, or non-allergens, for example, cigarette/cooking smoke, burning garbage, and motor vehicle fumes. ${ }^{20}$

Asthma is often caused by allergies, so it is also known as allergic asthma. The special characteristics of allergic asthma include increased chronic inflammation of the respiratory tract accompanied by inflammatory cell infiltration, proinflammatory cytokine release, and lipid mediator secretion that stimulate excessive mucus production, hyperresponsiveness, remodeling, and various respiratory disorders..$^{21,22}$ Airway inflammation in asthma is Th2-mediated, which is an adaptive immune response. Increasing evidence indicates that the innate immune response plays an important role in the pathophysiology of asthma, that is, in stable chronic inflammation and during exacerbation periods of acute inflammation in response to various stimuli (e.g., inhaled allergens, microbial infections, and environmental pollutants). ${ }^{22}$ Allergic asthma equals eosinophilic bronchitis and is characterized by a strong type2 cytokine response involving, namely, IL-4, IL-5, and IL$13 .{ }^{23}$ More than $50 \%$ of patients with eosinophilic allergic asthma develop an excessive type-2 immune response to inhaled allergens, including HDM allergens. ${ }^{24}$

\section{House dust mite allergens}

House dust mites, including Dermatophagoides pteronyssinus and Dermatophagoides Farinae, cause many kinds of allergies in humans, including allergic rhinitis, rhino-conjunctivitis, asthma, and atopic dermatitis. ${ }^{25}$ The allergenicity of HDM is contained in the mites, their eggs, carcasses, and fecal pellets. ${ }^{10,26,27}$ HDMs produce fecal particles and partially digested enzymes that are covered in dust particles throughout their life. ${ }^{25}$ Fecal pellets are composed of three to five balls containing debris, food, and proteolytic enzymes accumulated in mucus, encased in a chitin (a glucosamine-based polymer) peritrophic membrane, and expelled from the mite. The average diameter of the whole fallen mite is about $10-40 \mathrm{~mm}$ and, thus, can be inhaled and deposited in the respiratory tract. Mite exoskeletons, formed from chitin, can also stimulate an immune response. ${ }^{10}$

HDMs contain various allergens, including immunogenic epitopes, proteases, polysaccharide chitin,

Table 1. Characteristic differences of allergic and non-allergic asthma. ${ }^{19}$

\begin{tabular}{ll}
\hline \multicolumn{1}{c}{ Allergic Asthma } & \multicolumn{1}{c}{ Non-Allergic Asthma } \\
\hline Eosinophilia detected in blood & Normal eosinophilia count in blood \\
Eosinophilia detected in sputum & No eosinophils in sputum \\
Elevation of total IgE level & Normal total IgE level \\
Elevation of antigen-specific IgE & No antigen-specific IgE detected \\
$\begin{array}{l}\text { Pathological condition: Obstruction in respiratory, } \\
\text { hypertrophy of mucous glands, and eosinophil infiltration }\end{array}$ & \\
\hline
\end{tabular}


and lipopolysaccharides (LPS), that can trigger innate and adaptive immunity and induce a strong type- 2 immune response (Figure 1). ${ }^{25}$ The highest concentration of HDM allergens is found in their feces, but several studies have shown that HDM allergens can also be carried on various other particles. ${ }^{28}$ HDM allergens, together with nonallergenic substances, are a substantial cause of Th2 responses that induce $\mathrm{IgE}$ antibodies. HDM allergens and their adjuvant effects can increase IgE sensitization. ${ }^{26}$

There are more than 30 IgE-inducing HDM allergen groups possessing different allergenicity; they are categorized as serodominant, mid-tier, minor, and unknown importance allergens. ${ }^{29,30}$ Serodominant indicates the most dominant allergen contributing to the IgE response quantitatively, including groups 1,2, and 23. Mid-tier includes groups $4,5,7$, and 21 , whereas minor includes groups $3,6,8,9,10,11,13,15,16,17,18$, and 20. Groups 14, 22, and 24-33 are considered unknown importance allergens. The group number order is based on IUIS allergen nomenclature, which refers to the prevalence and not the severity of allergy manifestation. ${ }^{29}$

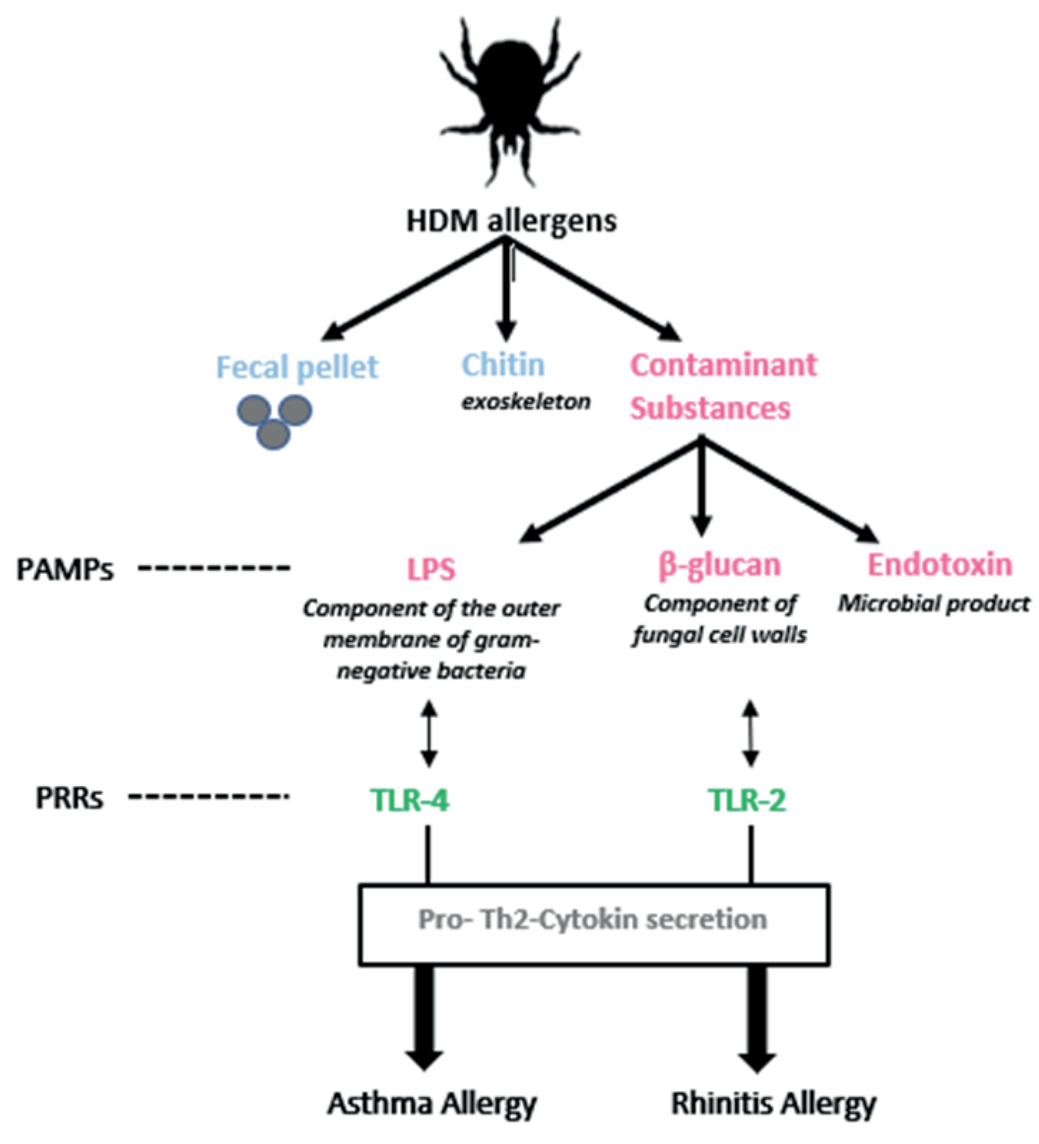

The allergenicity of HDM does not depend on the HDM viability. Several HDM-specific allergens have been characterized and isolated (Table 2). ${ }^{26,28}$ Group 1 (Der $p$ 1 and $\operatorname{Der} f 1$ ) and group $2(\operatorname{Der} p 2$ and $\operatorname{Der} f 2$ ) HDM allergens are considered the most immunodominant HDM allergens. ${ }^{26,28}$ The HDM-specific allergens, Der $p 1$ and Der $p 2$, are derived from Dermatophagoides pteronyssinus; Der $f 1$ and $\operatorname{Der} f 2$ derive from Dermatophagoides farinae. ${ }^{28}$ The immunostimulant effect comes from the HDM allergen itself. In group 1, the main allergen is a cysteine protease that increases the respiratory epithelium permeability via enzymatic digestion of tight junctions. Proteolytic activity initiates transepithelial allergen delivery by disrupting tight junctions, cleaving the CD23 and CD2 differentiation clusters and, thereby, inducing IgE synthesis. Alternatively, in group 2, the allergen is homologous to the MD-2 adapter protein (TLR co-receptor) that facilitates LPS-mediated TLR-4 signaling. HDM particles contain PAMPs (including the DNA of mites and bacteria) and endotoxins, which can stimulate the innate immune response causing allergic reactions. ${ }^{26,28}$
Figure 1. HDM contaminant-allergen components causing airway allergy. ${ }^{10,32,34}$ HDM contaminant allergens include LPS, $\beta$-glucan, and endotoxin. LPS and $\beta$-glucan may promote pro-Th2-cytokine secretion through TLR-4 and TLR-2 activation, respectively. 
Table 2. Several HDM allergen groups and mechanisms of allergenicity. ${ }^{10,26}$

\begin{tabular}{cll}
$\begin{array}{c}\text { Allergens } \\
\text { Group }\end{array}$ & Component & Mechanism of Allergenicity \\
\hline 1 & Cysteine protease & Obstruction of airway and disruption of epithelial tight junction \\
\hline 2 & Lipid-binding protein (MD2-like) & MD2 molecular mimicry, activation of DCs through TLR-4 and TLR-2 \\
\hline 3 & Serine protease (trypsin-like) & Disruption of tight junctions, activation of PAR-2 in keratinocytes and airway epithelium \\
\hline 5 & Lipid binding protein & Possibly bind hydrophobic ligands stimulating innate immunity \\
\hline 6 & Serine protease (chymotrypsin-like) & Disruption of tight junctions, activation of PAR-2 in keratinocytes \\
\hline 7 & Lipid binding protein & Lipid-binding protein molecular mimicry, activation of DCs via TLR-2, TLR-3, and TLR-4 \\
\hline 9 & Collagenolytic protease & Disruption of tight junctions, activation of PAR-2 in keratinocytes and airway epithelium \\
\hline
\end{tabular}

Recently, HDM allergens have been identified that can induce an innate immune response. In HDM allergy, when a person is exposed to an allergen, PRRs from innate immune cells recognize PAMPs. ${ }^{31}$ HDM-induced innate immunity activation causes an inflammatory response in the respiratory tract through various receptors, such as TLR-2, TLR-4, Dectin-1, and Dectin-2. Additionally, HDM allergen exposure causes $\mathrm{CD}^{+}$memory $\mathrm{T}$ cells (HDM allergenspecific) to stimulate an inflammatory response in tissues as well as induce Th17 and Tfh cells ( $\mathrm{T}$ follicular helper) in allergic airway inflammation. ${ }^{25}$

\section{Innate immune response against HDM- induced allergic asthma}

Various experiments have proven that HDM allergenspecific Th2 cells are the primary players in the immune response to HDM; as such, adaptive immune responses are considered the most important responses to HDM allergens. ${ }^{32}$ However, to date, there is more evidence showing that the innate immune response is equally important in the pathophysiology and protection against asthma. ${ }^{11,21}$ The innate immune system plays an important role in determining the adaptive immune response. ${ }^{15}$

HDM allergens induce Th2 immunity through stimulation of different innate immune pathways (Figure 2). For example, respiratory epithelial cells express PRRs that can detect environmental stimuli. Besides, PRRs can also release endogenous danger signals, resulting in DC activation that links the innate and adaptive immunity. ${ }^{21}$ There are several pathways of the innate immune response to HDM allergens. Specifically, protease allergens cleave the protease-sensitive receptor (PAR)-2. HDM allergens damage the epithelial barrier to gain access to DCs and damage epithelial tissue in order to trigger the tissue release of "danger signals" or damage-associated molecular patterns (DAMPs), such as ATP (adenosine triphosphate) and uric acid. Furthermore, HDM allergens contaminated with PAMPs of other microbes can activate PRRs and release DAMPs. The glycan component of the HDM can activate DCs through the CLRs. ${ }^{32}$

These signaling pathways lead to massively elevated cytokine levels from the innate immune response (chemokines), including IL-1 $\beta$, IL-6, TSLP, IL-25, IL-33, CCL20, or GM-CSF. These cytokines can stimulate the recruitment and activation of inflammatory cells, as well as the induction of Th2 differentiation. Expression of OX40L in DCs and IL-4 in basophils is mediated by TSLP and initiates Th2 polarization responses. IL-25 and IL-33, stimulated by allergen binding to C-type lectin receptors (CLR), are potent activators of ILC-2. Activated ILC-2 stimulates the release of IL-5 and IL-13 cytokines. These two cytokines provide the main characteristics of allergic inflammation in the respiratory tract, including IgE secretion following B-cell activation, remodeling of the respiratory tract, and infiltration of eosinophils into areas of inflammation. ${ }^{26,32}$

Allergic reactions induced by HDM, including allergic rhinitis, asthma, and atopic and contact dermatitis, are mostly type I hypersensitivity reactions. When HDM allergens enter the respiratory system, they are recognized and endocytosed by DCs beneath the airway epithelium. Inhaled HDM allergens cause eosinophil recruitment to 


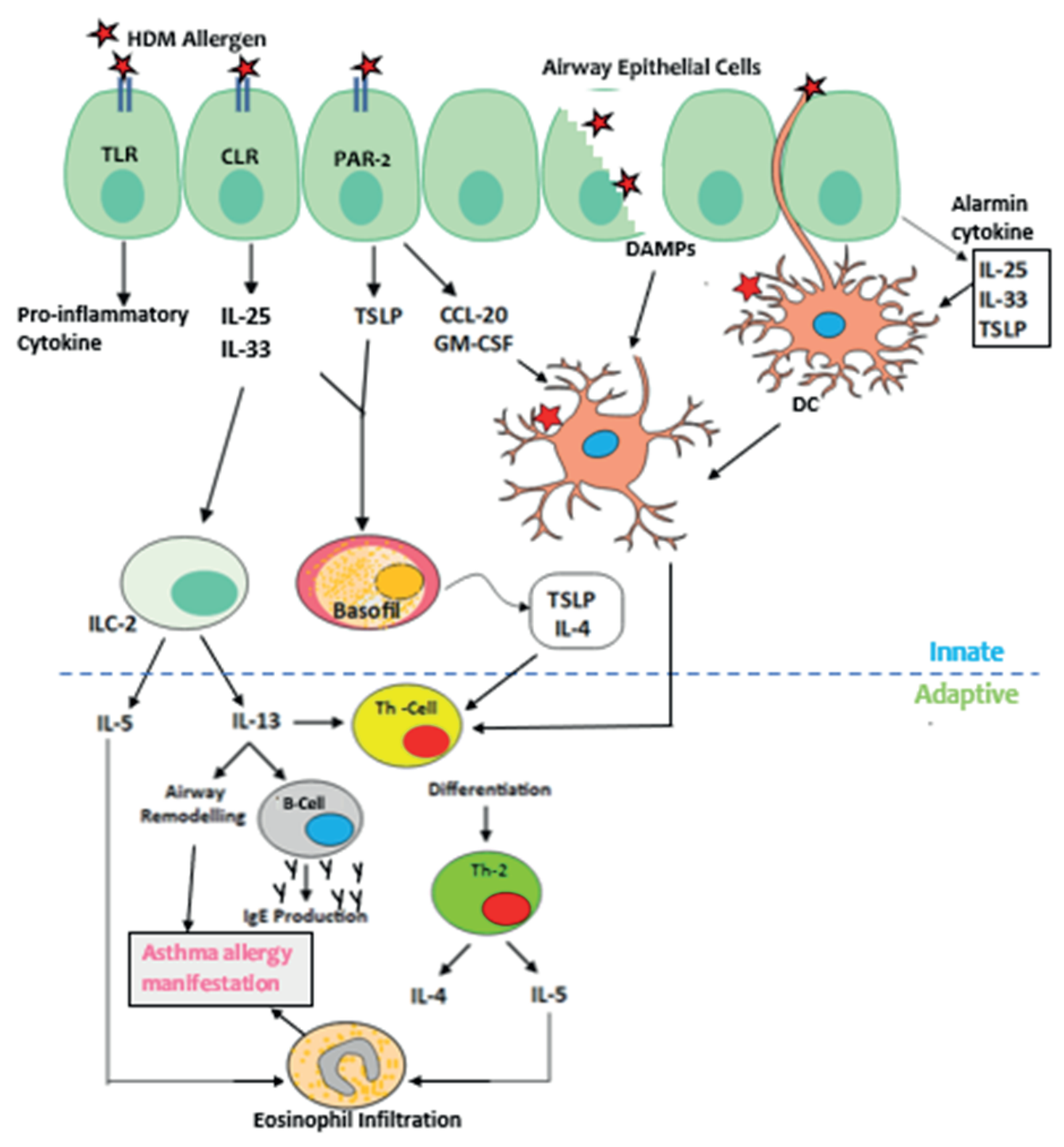

Figure 2. Immune response mechanism against HDM allergens causing asthma allergy. ${ }^{32}$ There are several pathways of the innate immune response to HDM allergens. HDM allergens recognized by PRRs in airway epithelial cells (TLR, CLR, PAR-2) causing pro-inflammatory cytokines and chemokines release, including IL-1 $\beta$, IL-6, TSLP, IL-25, IL-33, CCL20, and GM-CSF. HDM allergens damage the epithelial barrier to gain access to DCs and damage epithelial tissue to trigger the tissue release of DAMP. These signaling pathways induce ILC-2 activation and Th2 differentiation leading to the release of IL-5 and IL-13 cytokines. These two cytokines promote remodeling of the respiratory tract, IgE secretion, and infiltration of eosinophils which are asthma allergy manifestations.

the lungs and DCs; simultaneously, IL-4 from basophils activates the inflammatory pathway. Subsequently, the epitope is presented to $\mathrm{B}$ and $\mathrm{T}$ cells. ${ }^{10,27}$ Mucosal inflammation depends on innate and adaptive immune responses occurring in the epithelial cells of the respiratory tract. $^{10}$

HDM allergen sensitization is associated with prolonged (persistent) allergic asthma and decreased lung function in children. HDM sensitization caused by inhaled allergens occurs via the respiratory tract mucosa. ${ }^{26}$ Immunological mechanisms progress due to an aberrant Th2 response that activates cellular events (eosinophilia, isotype switching of B cells to produce IgE, mast cell degranulation, etc.) in target tissues. Disruption of the balance between Th1, Th2, and T-regulator (T-reg) effector cells underlies allergic reactions. ${ }^{33}$ 


\section{Innate immune components against HDM}

\section{Pattern Recognition Receptors (PRRs)}

PRRs are not only expressed on antigen-presenting cells (APCs; e.g., macrophages and dendritic cells), inflammatory cells (e.g., eosinophils and basophils), or non-professional immune cells (e.g., epithelial cells, endothelial cells, keratinocytes, and fibroblasts). Airway epithelial cells are the first-line barrier exposed to the environment, including inhaled air allergens that induce an allergic response. Epithelial cells express various PRRs (e.g., TLR-2, TLR-3, TLR-4, TLR-5, NODI-2, NLRP-3, Dectin-1, and PAR-2) in response to microbial PAMPs or DAMPs (damage-associated molecular patterns), which are released as 'danger signals' in the event of tissue damage, cellular stress, or even cell death. Persistent epithelial PRR activation-induced allergens sensitize Th2 cells to massively release proinflammatory cytokines, chemokines, growth factors followed by an increased influx of Th2 cells, basophils, eosinophils, DCs, and other inflammatory cells. ${ }^{32}$ (Figure 2).

Activated PRRs, upon recognition of PAMPs, induce signal transduction involving nuclear factor-kappa B (NF$\kappa B)$, interferon type 1 , and mitogen-activated protein kinase (MAPK), thereby increasing essential proinflammatory cytokines and chemokines in inflammation and antimicrobial responses..$^{34}$ Activation of NF- $\mathrm{KB}$ and interferon regulatory factors (IRF) 3 and 7 (due to the binding of TLRs) stimulate proinflammatory cytokines, chemokines, interferons, defensins, and prostaglandins production. ${ }^{21}$ Unlike other TLRs that require only one signal from the myeloid differentiation factor-88 (MyD88)/MyD88-adapter-like (MAL) molecule or the TIR domain-containing adapterinducing interferon- $\beta /$ TRIF-related adapter molecule (TRIF/TRAM) adapter pair, TLR-4 requires both MyD88/ MAL and TRIF/TRAM signals. Signaling pathways that use MyD88 lead to interferon production. ${ }^{16}$

When stimulated by the binding of TLRs and their ligands, MyD88 interacts with the cytoplasmic domain of TLR and recruits IL-1R-associated kinase-4 (IRAK-4) and IRAK-1, thereby binding the MyD88 death domain. IRAK1 and IRAK-4 then associate with ubiquitin ligase (E3)/ TNF receptor-associated factor-6 (TRAF-6). Furthermore, TRAF-6 cooperates with the E2 ligase complex (UBC13 and UEV1A) to catalyze the formation of K63-linked polyubiquitin chains at two sites-TRAF-6 and NF- $\kappa$ B essential modifier (NEMO) - thereby activating the IKK complex (I- $\kappa \mathrm{B}$ kinase). This ubiquitination process activates the TGF$\beta$-activated kinase 1 (TAK1) complex. ${ }^{13,16}$

Activated TAK1 phosphorylates and activates NEMO (IKK complex); then, IKK phosphorylates I-אB. Phosphorylated I- $\mathrm{B}$ will ubiquitinate K48-linked chains and degrade $\mathrm{I}-\kappa \mathrm{B}$ via NF- $\mathrm{BB}-$ releasing proteosomes. Translocation of NF- $\mathrm{KB}$ into the nucleus initiates the expression of proinflammatory cytokine genes, such as IL$1 \beta$, IL- 6 , and tumor necrosis factor- $\alpha(\mathrm{TNF}-\alpha)$. TAK1 also activates certain MAPKs, such as c-Jun terminal kinase (JNK) and MAPK p38, which also lead to the induction of cytokine genes through activation of transcription factor AP-1 (activator protein-1). ${ }^{13,16}$

In addition to carrying their own allergens, HDM is also a crucial transporter of microbial compounds because house dust also contains bacterial LPS and fungal $\beta$-glucan, which can be associated (contaminated) with HDM (Figure 1). LPS from gram-negative bacteria can cause allergic respiratory inflammation. LPS is the main PAMP that activates the HDMinduced innate immune response via TLR-4 in lung mucosa and allergic asthma. In HDM, there are also endosymbionts, as evidenced by bacterial $16 \mathrm{~S}$ ribosomal DNA. ${ }^{34}$ The TLR4-mediated response requires the regulated action of other soluble CD14 and TLR-4 cofactors, such as MD-2. ${ }^{21}$ TLR-4 in epithelial cells, triggered by LPS from HDMs, extracts and stimulates the pro-Th2 cytokine secretion of TSLP, IL-25, IL-33, and GM-CSF. Meanwhile, $\beta$-glucan from HDMs is the main PAMP that activates innate immunity through TLR-2 in the nasal mucosa and triggers allergic rhinitis. Endotoxins, which are the main microbial products, can also contaminate HDM allergens. Endotoxin levels in house dust are inversely related to allergen sensitization and atopic asthma development. Epidemiological studies and mouse models show that the LPS dose determines the allergic response. Inhaled low-dose LPS can stimulate a Th2 response to develop antigen sensitization and eosinophilic inflammation. In contrast, high-dose LPS induces a Th1 response that decreases Th2-type cytokines, thereby preventing eosinophilic inflammation. ${ }^{32}$

\section{Proteinase-Activated Receptors-2 (PAR-2)}

PARs are activated by serine proteases through the proteolytic cleavage of the extracellular N-terminus. PARs play a role in innate and protective immune responses through their ability to detect extracellular microbial proteases. The inflammatory response to microbial proteases is related to the role of PAR-2. TLR- 4 cooperates with 
PAR-2 in terms of signal transduction. ${ }^{32}$ Additionally, PAR2 signals play a role in modulating immune mediators in respiratory epithelial cells that contribute to allergic asthma manifestation. ${ }^{35}$

Cysteine proteases in HDM allergens can reduce epithelial cell tight junctions and activate PAR-2, resulting in E-cadherin disruption at intercellular contacts. As a result, allergens can reach submucosal cells and increase allergic inflammation. Activated PAR-2 can induce intracellular signaling activity, including $\mathrm{NF \kappa B}$ and extracellular kinase signaling pathways that increase the proinflammatory cytokine IFN- $\gamma .8$ Exogenous proteases that react with PAR2 also cause leukocyte infiltration and increase response to allergens. PAR activation on eosinophils and mast cells stimulates their degranulation (Figure 2). Animal studies indicate that PAR-2 activation may exacerbate chronic airway inflammation. Asthmatic patients showed increased PAR-2 in epithelial cells compared to healthy control individuals. ${ }^{10}$

\section{Dendritic Cells (DCs)}

Dendritic cells (DCs) are the primary cell type responsible for sensitization and induction of effectors in allergy. Allergen exposure to DCs causes DC migration from peripheral sites, specifically, lungs to the lymph nodes, to stimulate naive $\mathrm{T}$ cells differentiation into allergen-specific Th2 cells and be accompanied by allergic inflammation of the respiratory tract. ${ }^{2,32}$ DCs initiate Th2 differentiation by releasing innate proinflammatory cytokines, including TSLP, IL-25, IL-33, and GM-CSF (Figure 2). ${ }^{10}$ Therefore, DCs can be an important target for therapeutic interventions in controlling allergic respiratory diseases. ${ }^{2}$

The effector function of DCs is initiated when an allergen epitope is presented to both $\mathrm{T}$ and $\mathrm{B}$ cells, causing stimulation of Th2 cells to secrete various cytokines and B cells to secrete specific IgE antibodies. IgE on mast cell and basophil receptors bind to allergen polymers with high affinity, causing mast cell degranulation then releasing inflammatory factors, such as histamine. Clinical manifestations include allergic rhinitis and difficulty (shortness) of breathing. Even if the allergic mechanism and pathway induced by allergens are generally the same, HDM allergen proteins have a 'cutting' effect on respiratory epithelial cells due to their enzymatic activity and unique three-dimensional spatial structure. These unique features of HDM allergens cause them to be bound by PRRs on epithelial cells, affecting the innate immune system. ${ }^{27}$

\section{Innate Lymphoid Cells-2 (ILC-2)}

ILC-2 represents group 2 of type 2 immune cells and is a major source of cytokines triggering allergic asthma. ${ }^{36,24}$ ILC-2 releases cytokines in response to "alarmin" cytokines released by epithelial cells upon exposure to HDM allergens (Figure 2). ${ }^{37}$ Cytokines produced by ILC-2 associated with Th2, including IL-4, IL-5, and IL-13. ${ }^{38}$ ILC is an innate lymphoid-cell potential trigger of allergic asthma manifestations based on studies on mouse models and samples of asthmatic patients. ${ }^{24}$

In asthmatic patients, there is an increase in ILC-2 levels detected in blood and sputum. ${ }^{24}$ Increased levels of ILC-2 in peripheral blood in allergic asthma are the main source of type 2 cytokines. ${ }^{37}$ Increased ILC-2 levels in patients who suffer from both allergic asthma and allergic rhinitis are caused by exposure to specific allergens, with a greater contribution of ILC-2 to the incidence of allergic asthma than allergic rhinitis. This is proved by a previous study which showed that the prevalence of ILC-2 in the blood was greater in the allergic asthma group than the allergic rhinitis group and the control group (healthy). ${ }^{39}$

ILC-2 is found in several organs and tissues, including the brain, heart, liver, skin, intestines, and lungs. ${ }^{37}$ ILC2 is thought to control homeostasis in the lungs, small intestine, and peripheral blood. ILC-2 in the lung indicates its involvement in the pathophysiology of allergic inflammation and asthma. ${ }^{40}$ ILC-2 plays a role in regulating respiratory tract inflammation in eosinophilic asthma, regardless of its association with atopic factors in asthmatic patients and adaptive immunity. ${ }^{37,38}$ This is due to the absence of specific antigen receptors on ILC-2 cells. However, ILC-2 responds to signals from the epithelium through the mediation of "alarmin" cytokines, namely IL-25, IL-33, and TSLP produced by the epithelial cells themselves. ILC-2 and basophils can cause eosinophilic inflammation in some asthmatic patients, so it is suspected that ILC-2 is involved in allergic asthma and asthma exacerbations. ${ }^{37,38}$

\section{Mediators: CCL-20, GM-CSF, TSLP, IL-25, IL-33, IL-5, and IL-13}

The stimulated airway epithelium, for example by HDM allergens, increases the production of the CCL-20 chemokine, which attracts DCs to the tissue. ${ }^{16}$ HDMs can bring spores of fungi on their exoskeletons; fungi originally live in the HDM gut. C-type lectin receptors (CLRs), namely Dectin-1 and Dectin-2, expressed on myeloid cells, 
act as PRRs that recognize $\beta$-glucans found in fungal and bacterial cell walls. CLRs and $\beta$-glucan binding bridge the innate and adaptive immune responses. Airway epithelium exposed to HDM allergens cause $\beta$-glucan-dependent CCL20 secretion. $^{10}$

IL-33 is a growth factor for ILC-2, especially pulmonary ILC-2, and stimulates the differentiation, maturation, and expression of type- 2 cytokines. ${ }^{36} \mathrm{IL}-33$ is produced by activated mast cells and plays an essential role in enhancing the Th2 response. ${ }^{16}$ IL-33 and pulmonary TSLP are produced mainly in respiratory epithelial cells. ${ }^{36}$ TSLP, IL-25, and IL-33 have a major role in the pathogenesis of asthma by promoting type- 2 immune cells, namely ILC- 2 and Th2, to release IL-4, IL-5, and IL-13. Increased levels of these three cytokines are a marker of air pollutants, microbes, or allergens that enter the respiratory epithelium. These particles activate ILC- 2 and Th2 to release various type-2 cytokines, ultimately manifesting as eosinophilia, immune pathology, hyperresponsiveness, and airway remodeling. ${ }^{41}$ IL-5 produced Th2 plays a role in recruiting and activating eosinophils. ${ }^{16}$ IL-13 levels are increased in atopic asthma compared to non-atopy. IL-13, which is a proinflammatory Th2 cytokine, can be a marker of chronicphase inflammatory reactions. ${ }^{8}$

GM-CSF stimulates neutrophil production. ${ }^{12}$ When epithelial cells of asthmatic patients are cultured, there is a continuous over-production of GM-CSF, suggesting that GM-CSF plays a role in regulating asthma. The same thing was also seen from in the bronchial and sputum biopsies of severe asthma patients, an increase in TSLP. Genetic polymorphisms in the promoter region of TSLP are associated with an increased risk of developing asthma. TSLP stimulates bronchial epithelial cell proliferation and IL-13 production. ${ }^{21}$ TSLP is fcytokine for the initiation and maintenance of Th2 allergic inflammation; it is also a susceptibility asthma gene in various ethnicities. The function of TSLP is innate and not specific to any particular allergen. TSLP causes asthma by promoting an allergic innate immune response to HDM allergens leading to Th2 inflammation in the respiratory tract. ${ }^{42}$ TSLP binds to a surface heterodimer receptor called TSLPR (thymic stromal lymphopoietin receptor), which is expressed on many immune cells, including T cells, B cells, natural killer (NK) cells, monocytes, basophils, eosinophils, ILC-2, and DCs. ${ }^{38}$ Both GM-CSF and TSLP stimulate DCs to develop type-2 immune responses. ${ }^{21}$

\section{Therapeutic potency of innate immune components}

Since asthma is a complex disease with heterogeneous inflammatory pathways, personalized treatment options become more relevant for asthmatic patients. An endotypes approach based on an anti-allergen immune mechanism is one such specific therapy, especially for "severe" patients who do not react to standard anti-inflammatory medications (inhaled corticosteroids) or bronchodilators. ${ }^{38}$ Treatments for allergic asthma based on the components of the adaptive immunity have been developed and licensed, such as monoclonal antibodies $(\mathrm{mAb})$ that target $\mathrm{IgE}$, Th2 cytokines, or their receptors. However, their development is limited due to the high cost; as such, breakthroughs are needed to overcome these problems. Currently, new drugs are being developed that target the upstream mediators of the innate immune system. An understanding of the innate immune response and its components in allergic asthma caused by HDM allergens has the potential to identify new drug targets and be an alternative to previous drugs, such as TLR-4 agonists, anti-TSLP, anti-IL-5, and chemoattractant receptor-homologous molecule expressed on Th2 cells (CRTh2) antagonists. ${ }^{21,31,37}$

TLR-4 agonist immunotherapy was developed from 1D10G (1-dehydro-10-gingerdione), a pungent substance isolated from ginger (Zingiber officinale). These substances can inhibit the TLR-4-mediated signaling pathway from activating NF- $\mathrm{kB}, \mathrm{AP}-1$, or IRF-3 as well as inflammatory cytokine gene expression in LPS-activated macrophages by blocking LPS binding to MD-2 (myeloid differentiation factor-2). SNP's (single nucleotide polymorphism's) MD2 gene in the proximal promoter regions (i.e., rs1809441 and rs1809442) is over-expressed in HDM allergic patients. In their serum, SNPs correspond with high IgE levels specific to $\operatorname{Der} p 1$ and $\operatorname{Der} p 2$. Specific MD2 immunotherapy is a new alternative for inhibiting the effects of HDM allergen exposure (especially from the Der $p 2$ group) on the manifestations of allergic inflammation. ${ }^{31}$

The expression of epithelial cytokines is modified due to exposure to environmental allergens and is often associated with asthma exacerbations; thus, cytokines, such as IL-25, IL-33, and TSLP, are potential targets for future asthma treatment. ${ }^{41}$ The three "alarmin" cytokines, which are HDM allergen-selective protease inhibitors, and their receptors are also potential targets for the treatment 
of asthma and other allergies. ${ }^{10}$ Currently, phase 2 trials are underway on IL-33 as a potential therapeutic target. The antiIL-33 mAb, REGN3500, has shown efficacy (compared to a placebo and asthma control groups) in asthma treatment, characterized by improved lung function. ${ }^{41}$

Anti-TSLP interventions may be useful for asthmatic patients who do not respond to anti-IL-33 receptor therapy. ${ }^{36} \mathrm{An}$ anti-TSLP, in the form of a human IgG2 mAb named Tezepelumab, is being researched and developed specifically to inhibit TSLP cytokines. When Tezepelumab binds to the TSLP protein, the interaction between TSLP and its receptor complex is blocked. As a result, there is a decreased effect on APC recruitment and maturation of adaptive immune cells, resulting in inhibition and suppression of type 2 inflammation. ${ }^{38,43}$ One study's clinical trial revealed decreased blood eosinophil counts, IgE levels, and FeNO concentrations after 4 weeks of Tezepelumab administration. Asthmatic patients given Tezepelumab showed a clinically significant reduction in the exacerbation period and improved lung function. ${ }^{38}$ Tezepelumab also reduced airway inflammation and bronchoconstriction in asthmatic patients in pre- and post-allergen exposure trials. However, Tezepelumab is still in a phase 3 study evaluating its efficacy and safety in severe adult asthma patients and has not yet been approved. ${ }^{38,41}$

New biologic drugs (e.g., Mepolizumab and Reslizumab) targeting IL-5 are an alternative therapy for patients with severe eosinophilic asthma. This cytokine, as the main activator of eosinophils, was chosen since it plays a pivotal role in determining and maintaining eosinophilic inflammation in all phenotypes 2.38,43 Mepolizumab is a $\mathrm{mAb}$ that targets circulating IL-5, decreasing the proliferation, maturation, and survival of eosinophils. Mepolizumab and Reslizumab, at a fixed dose of $300 \mathrm{mg}$ every 4 weeks subcutaneously, are effective in preventing asthma exacerbations. ${ }^{38}$ Mepolizumab and Reslizumab bind IL-5, thereby preventing binding to the IL-5 receptor (IL-5R) on eosinophils. This subsequently decreases the inflammation caused by the recruitment and activation of eosinophils. In contrast, Benralizumab works by binding directly to IL-5R $\alpha$ on eosinophils, thereby preventing IL-5 binding, and stimulates eosinophil cell death through an ADCC (antibody-dependent cell-mediated cytotoxicity) mechanism. ${ }^{41}$

CRTh2 expressed on eosinophils, mast cells, and basophils bind prostaglandin D2 and contribute to allergic inflammation and eosinophil activation. CRTh2 activation has cellular effects, such as chemotaxis, Th2 cytokine production by Th2 cells and ILC2, eosinophil cationic protein $(\mathrm{ECP})$ release from eosinophils, and allergen-induced histamine release from basophils. CRTh2 antagonists are in clinical development for asthma treatment. Oral CRTh2 antagonists provided airway function improvement and protection against symptoms in asthma patients in clinical trials. ${ }^{44,45}$

\section{Conclusion}

The role of adaptive immunity, especially that carried out by Th2 in allergic asthma due to HDM allergen exposure, is the main mechanism of concern. However, recent studies have shown that the role of innate immunity is also vitally related to allergic asthma incidence. In the adaptive immune response, Th2 is activated and functions due to the initiation and stimulation of several innate immune response pathways, including the breakdown of PAR-2 by HDM protease activity, which stimulates several triggers of allergic asthma manifestations. By disrupting the epithelial barrier, the HDM-induced innate immune response stimulates DC activation. Besides, activation of PRRs (TLRs and CLRs) due to allergen binding triggers signaling pathways that produce proinflammatory cytokines. All of these pathways lead to allergic asthma manifestations. Understanding the innate immune response to HDM allergens is a promising source for the development of alternative therapeutic targets based on the immune components involved in allergic asthma; for example, anti-TSLP, anti-IL-5, and specific MD-2 immunotherapies.

\section{References}

1. Oemiati R, Sihombing M, Qomariah. Faktor-faktor yang berhubungan dengan penyakit asma di Indonesia. Media Peneliti dan Pengembangan Kesehatan. 2012; 20(1): 41-50.

2. Voskamp AL, Kormelink TG, Hiemstra PS, Taube C, Jong EC De, Smits HH. Modulating local airway immune responses to treat allergic asthma: lessons from experimental models and human studies. Semin Immunopathol. 2020; 42(1): 95-110.

3. Andriani FP, Sabri YS, Anggrainy F. Gambaran karakteristik tingkat kontrol penderita asma berdasarkan indeks massa tubuh (IMT) di Poli Paru RSUP. Dr. M. Djamil Padang pada tahun 2016. Jurnal Kesehatan Andalas. 2019; 8(1): 89-95.

4. Kemenkes RI. Hasil Utama Riset Kesehata Dasar (RISKESDAS). Jakarta: Kemenkes RI; 2018.

5. Kowal K, Pampuch A, Siergiejko G, Siergiejko Z. Sensitization to major dermatophagoides pteronyssinus allergens in house dust mite allergic patients from North Eastern Poland developing rhinitis or asthma. Adv Med Sci. 2020; 65(2): 304-9. 
6. Thomas WR, Hales BJ, Smith W. House dust mite allergens in asthma and allergy. Trends Mol Med. 2010; 16(7): 321-8.

7. Erban T, Klimov P, Talacko P, Harant K, Hubert J. Proteogenomics of the house dust mite, Dermatophagoides farinae: Allergen repertoire, accurate allergen identification, isoforms, and sex-biased proteome differences. J Proteomics. 2020; 210: 103535. doi: 10.1016/j. jprot.2019.103535.

8. Kwarta CP, Wibowo H, Khaedir Y, Rengganis I, Nuraeni HS. Interleukin-13, interleukin-10, interferon-g and IDO production in response to home dust mite in allergic asthma. Indones Biomed J. 2019; 11(2): 194-9.

9. Calderon MA, Linneberg A, Hernandez D, Rojas F De, Virchow JC, Demoly $\mathrm{P}$, et al. Respiratory allergy caused by house dust mites : What do we really know? J Allergy Clin Immunol. 2015; 136(1): $38-48$.

10. Gregory LG, Lloyd CM. Orchestrating house dust mite-associated allergy in the lung. Trends Immunol. 2011; 32(9): 402-11.

11. Simpson JL, Brooks C, Douwes J. Innate immunity in asthma. Paediatr Respir Rev. 2008; 9(4): 263-70.

12. Abbas AK, Lichtman AH, Pillai S. Cellular and Molecular Immunology. 9th ed. Philadelphia: Elsevier; 2018.

13. Akira S, Uematsu S, Takeuchi O. Pathogen recognition and innate immunity. Cell. 2006; 124(4): 783-801.

14. Gasteiger G, D'osualdo A, Schubert DA, Weber A, Bruscia EM, Hartl D. Cellular innate immunity: an old game with new players. J Innate Immun. 2017; 9(2): 111-25.

15. Finn PW, Bigby TD. Innate immunity and asthma. Proc Am Thorac Soc. 2009; 6(3): 260-5.

16. Murphy K. Janeway'S Immunobiology. 7th ed. New York: Garland Science; 2008

17. Virella G. Overview of hypersensitivity. In: Virella G, editor. Medical Immunology. 7th ed. Boca Raton: Taylor \& Francis Group; 2020. p.277-86.

18. Kurnia FN, Hartana A, Rengganis I. Faktor pencetus kejadian alergi pernapasan pada pasien dewasa di RSUPN Dr. Cipto Mangunkusumo. Jurnal Sumberdaya HAYATI. 2019; 5(2): 72-80.

19. Finn AF, Virella G. IgE-mediated (immediate) hypersensitivity. In: Virella G, editor. Medical Immunology. 7th ed. Boca Raton: Taylor \& Francis Group; 2020. p.287-301.

20. Dharmayanti I, Hapsari D, Azhar K. Asma pada anak di Indonesia : Penyebab dan pencetus.Jurnal Kesehatan Masyarakat Nasional. 2013; 9(29): 320-6.

21. Lambrecht BN, Hammad H. The airway epithelium in asthma. Nat Med. 2012; 18(5): 684-92.

22. Barnig C, Levy BD. Innate immunity is a key factor for the resolution of inflammation in asthma. Eur Respir Rev. 2015; 24(135): 141-53.

23. Borish L. The immunology of asthma: Asthma phenotypes and their implications for personalized treatment. Ann Allergy Asthma Immunol. 2016; 117(2): 108-14.

24. Stadhouders R, Li BWS, de Bruijn MJW, Gomez A, Rao TN, Fehling $\mathrm{HJ}$, et al. Epigenome analysis links gene regulatory elements in group 2 innate lymphocytes to asthma susceptibility. J Allergy Clin Immunol. 2018; 142(6): 1793-807.

25. Wada T, Hirahara K, Aoki A, Morimoto Y, Kiuchi M, Kumagai J, et al. An optimized protocol for the analysis of house dust mite (Dermatophagoides farinae) -induced neutrophil-dominant airway inflammation. J Immunol Methods. 2019; 465: 53-60.

26. Sánchez-borges M, Fernandez-caldas E, Thomas WR, Chapman MD, Lee BW, Caraballo L, et al. International consensus (ICON) on: Clinical consequences of mite hypersensitivity, a global problem.
World Allergy Organ J. 2017; 10(16): 1-26.

27. Cao H, Liu Z. Clinical significance of dust mite allergens. Mol Biol Rep. 2020; 47(8): 6239-46.

28. Adelman DC, Casale TB, Corren J. Manual of Allergy and Immunology. 5th ed. Philadelphia: Lippincott Williams \& Wilkins; 2012 .

29. Jacquet A. Characterization of innate immune responses to house dust mite allergens: pitfalls and imitations. Front Allergy. 2021; 2: 1-8. doi: 10.3389/falgy.2021.662378.

30. Thomas WR. Hierarchy and molecular properties of house dust mite allergens. Allergol Int. 2015; 64(4): 304-11.

31. Huang FL, Liao EC, Yu SJ. House dust mite allergy: Its innate immune response and immunotherapy. Immunobiology. 2018; 223(3): 300-2.

32. Jacquet A. Innate immune responses in house dust mite allergy. ISRN Allergy. 2013; 2013: 735031. doi: 10.1155/2013/735031.

33. Pacciani V, Gregori S, Chini L, Corrente S, Chianca M. Induction of anergic allergen-specific suppressor $\mathrm{T}$ cells using tolerogenic dendritic cells derived from children with allergies to house dust mites. J Allergy Clin Immunol. 2010; 125(3): 727-36.

34. Jacquet A. The role of innate immunity activation in house dust mite allergy. Trends Mol Med. 2011; 17(10): 604-11.

35. Nadeem A, Al-Harbi NO, Ahmad SF, Ibrahim KE, Alotaibi MR, Siddiqui N, et al. Protease activated receptor-2 mediated upregulation of IL-17 receptor signaling on airway epithelial cells is responsible for neutrophilic infiltration during acute exposure of house dust mite allergens in mice. Chem Biol Interact. 2019; 304: 52-60.

36. Verma M, Liu S, Michalec L, Sripada A, Gorska MM, Alam R. Experimental asthma persists in IL-33 receptor knockout mice because of the emergence of thymic stromal lymphopoietin-driven IL-9 + and IL-13 + type 2 innate lymphoid cell subpopulations. J Allergy Clin Immunol. 2018; 142(3): 793-803.

37. Kabata H, Moro K, Koyasu S, Asano K. Group 2 innate lymphoid cells and asthma. Allergol Int. 2015; 64(3): 227-34.

38. Khalaf K, Paoletti G, Puggioni F, Racca F, De Luca F, Giorgis V, et al Asthma from immune pathogenesis to precision medicine. Semin Immunol. 2019; 46: 101294. doi: 10.1016/j.smim.2019.101294.

39. Scadding GK, Scadding GW. Innate and adaptive immunity: ILC2 and Th2 cells in upper and lower airway allergic diseases. J Allergy Clin Immunol Pract. 2021; 9(5): 1851-7.

40. Yu S, Kim HY, Chang YJ, Dekruyff RH, Umetsu DT. Innate lymphoid cells and asthma. J Allergy Clin Immunol. 2014; 133(4): 943-50.

41. Tan R, Liew MF, Lim HF, Leung BP, Wong WSF. Promises and challenges of biologics for severe asthma. Biochem Pharmacol 2020; 179: 114012. doi: 10.1016/j.bcp.2020.114012.

42. Iijima H, Kaneko Y, Yamada H, Yatagai Y, Masuko H, Sakamoto T, et al. A distinct sensitization pattern associated with asthma and the thymic stromal lymphopoietin (TSLP) genotype. Allergol Int. 2013; 62(1): 123-30.

43. Grayson MH, Feldman S, Prince BT, Patel PJ, Matsui EC, Apter AJ Advances in asthma in 2017: Mechanisms, biologics, and genetics J Allergy Clin Immunol. 2018; 142(5): 1423-36.

44. Oliver ET, Chichester K, Devine K, Sterba PM, Wegner C, Vonakis BM, et al. Effects of an oral CRTh2 antagonist (AZD1981) on eosinophil activity and symptoms in chronic spontaneous urticaria. Int Arch Allergy Immunol. 2019; 179(4): 21-30.

45. Singh D, Ravi A, Southworth T. CRTH2 antagonists in asthma: Current perspectives. Clin Pharmacol Adv Appl. 2017; 9: 165-73. 